\title{
The design of knowledge flow Mechanism within Virtual technology innovation team
}

\author{
Qin-qin Zhao \\ School of Education Science of Mianyang Normal University, Sichuan province, China, 621900 \\ morningddp@aliyun.com
}

Keywords: Virtual Technological Innovation Team (VTIT), Knowledge flows, Mechanism design

\begin{abstract}
Within the Virtual Technology Innovation Team (VTIT), knowledge sharing and flow is the key to success. Identifying the critical success factors to the knowledge flow mechanism of VTIT and taking targeted strategies is helpful to improve the efficiency of knowledge flows and knowledge creation. In this paper we will investigate how to promote internal knowledge sharing, knowledge flows and knowledge interaction in VTIT, through the knowledge flow mechanism design objectives, design content, member selection, the formation of mode selection, team development agreement, to improve the team's overall competitiveness.
\end{abstract}

\section{Introduction}

Virtual technology innovation team, which is a cross-regional, cross-border scientific research organization, is the product of the Information Age ${ }^{[1]}$. The emergence and development of the VTIT is on behalf of the organization in the form of new trends in scientific research and technological innovation and new trends in virtual teams. As an effective organization in the form of collaborative innovation, VTIT demonstrates integration of resources, which reflects the obvious advantages of innovative task completion as well as scientific and technological promotion. Virtual Technology Innovation Technology Innovation team is based on tasks, with a common goal, complementary advantages, resource sharing, and the use of electronic information technology, across the barriers of time, space and organizational boundaries ${ }^{[2]}$; and is proved to be major opportunities for the integration of innovative research group advantages forces ${ }^{[3]}$.

First, IT connects different global research institutions and organizations together quickly, and captures the latest development in science and technology while quickly aware of new scientific information and technology growth. Second, Each independent research team is ability to get the qualifications of VTIT with the most advantage of resources, it is possible that each function and process are all the world's leading and sufficient to cover the entire process of scientific research. Once again, The VTIT partnership is based on various research tasks ${ }^{[4]}$. The VTIT disbanded after the issue is completed, which can reduce the resources waste and equipment idle ${ }^{[5]}$.

The above-mentioned characteristics of VTIT bring challenges to knowledge flows and knowledge management ${ }^{[6]}$. In fact, information asymmetry between the team members decides whether knowledge sharing can be effectively carried out or not, VTIT is composed of many elements, such as human, financial, material and other data, news, cases, instructions, etc. It is difficult for team members to assess the resources, capabilities and interest goals due to asymmetric information, these problems we mention above increase the barriers of knowledge sharing, and increase the difficulty of knowledge flow mechanism design.

\section{The mechanism design of knowledge flow within the VTIT}

Systems theory believes that the mechanism is the interaction between the various elements of the system, and constraint the form and movement principle reasonable in order to make the system as a whole virtuous cycle. It is the sum of the healthy development of the rules, procedures and machinery. It contains two meanings ${ }^{[7]}$ : (1) mechanism of action occurs automatically according to certain rules and lead to certain results. (2) mechanism is not the end result, not the initial cause, but hope into action. Visible virtual technology innovation team knowledge flow mechanism refers to the virtual technological innovation within the team to ensure knowledge sharing among 
members of the various elements and organic interaction, interaction and mutual promotion or restriction of basic principles, rules, methods, and supporting frame assemblies. Here, if we see the process of virtual technology innovation knowledge flows among team members as a system, then the knowledge flow mechanism refers the sum of the various systems to promote its effective knowledge.

A brief description of the formation process of VTIT: First, consider the current background and geographical environment by relying on the successful formation of a team of university innovation premise. Second, consider the management and team leader relationship. The staff of the university's administration and academic leaders have scientific status of a relatively clear knowledge and understanding, we should cherish the attitude of service for researchers to work; team leader is often the subject of academic leaders, academic skills while also familiar with the process of scientific research, research management department familiar with the work program, reducing the time spent on administrative examination and approval. Again, consider the relationship between fund raising and project application. Research management department is not only responsible for the management and use of research funding, but also to organize related disciplines, units, organized to raise funds for the college of human innovation team success.

Based on the above analysis, the first is to make sure the purpose, subject, condition, time, place and procedures, and then to form an integrated system with human, material and financial resources. In simple terms, the process of formation of is: the formation of the body (research management and team leader) under the premise of a clear purpose established to mobilize resources (human, material and financial resources) at the right time and place, the use of innovative theory and team theory of the formation of the program, according to the various factors combining reasonable logic to produce a smooth functioning entity organization.

The stability of VTIT is vulnerable. It is not enough to have the cooperation projects only. It also requires the common goals and obligations of both parties. Clear agreement can establish stable expectations, conducive to coordinate the interests of all parties, to eliminate the difficulties and barriers, to maintain long-term continuous learning. Agreement is a very important aspect of rights and obligations. It is needed to clearly define the respective responsibilities and to enjoy the benefits to be borne, and how cooperation should put their tangible and intangible resources, how to share the results. In the course of negotiations, the parties should be sufficient communication between research entities, to identify possible problems in the future knowledge-sharing as much as possible, and in accordance with their respective strengths and weaknesses to develop more appropriate protocols. The agreement should include tasks, sharing and distribution of benefits and risks of the dissolution of the terms of several main parts.

\section{Interface management within virtual technology innovation team}

(a) A knowledge management system

Knowledge management (KM) is the process of capturing, developing, sharing, and effectively using organisational knowledge. It refers to a multi-disciplined approach to achieving organisational objectives by making the best use of knowledge. It is essential to build a knowledge management system. The knowledge management system of VTIT consists of the following subsystems ${ }^{[8]}$ :

(1) Knowledge Mining: knowledge mining is that changing the tacit knowledge into explicit knowledge, or digging a new valuable knowledge from explicit knowledge. The experts and some team members who are imagination and good at expression, take mutual exchange, induction and other methods, and try to dig out the layers of tacit knowledge. Also, you can extract the rules, concept through information, data and text.

(2) Knowledge base: Knowledge base is a certain knowledge representation. It centralized storage the various fields of expertise within the team with the explicit knowledge and knowledge about the external environment, as well as the knowledge of experts, extraction and display nature of tacit knowledge. The knowledge base change the decentralized knowledge into gathering, categorized, processing and refining ones, and make it become a systematic, continuous development of knowledge assets and shared resources. 
(3) Knowledge Exchange interactive systems: interactive knowledge exchange system mainly consider how to facilitate the exchange of members on the tacit knowledge sharing and interaction, usually through the establishment of knowledge-sharing platform to achieve, interactive maps and electronic platforms, including discussion of knowledge systems. Most of tacit knowledge is hard-coded. The effective approach is to establish a "knowledge map", making team members to communicate face to face who are at two separate places, like a portrait. When they faced with the problem of non- explicit knowledge in their work, they can also use the electronic discussion system. The problems encountered can be hang on the electronic discussion boards, other members may be to respond promptly to the boards on the version of their experience and best practices, They can communicate skills , experience and know-how tacit knowledge anytime, anywhere. They can also create a variety of formal and informal communication "place”, such as regular or irregular held seminars, and expert information for members to communicate, to exchange experiences and skills; It would make people who have special knowledge and ability of some form compose various project teams, task forces, etc., so that members of the group exchange and sharing of knowledge in an interactive place, and create a variety of new knowledge.

(b) Incentives

Incentives are stimulation which is necessary to achieve knowledge sharing goals. Knowledge-sharing goals are inseparable from incentives. It is necessary to establish the appropriate incentive system in order to carry out knowledge sharing effectively.

(1) Performance incentives. Take the knowledge sharing factors into performance evaluation system, by way of an economic incentive in return. The risks and the effort to get gain are proportional to its commitments. This way make members more aware of the value of knowledge sharing and also help to improve the members' initiative to knowledge sharing.

(2) Prestige incentives. Reputation and prestige is a key motivating factor in knowledge sharing. It is necessary to establish the prestige or recognition in return, because they allow the members who feel achieve self-fulfillment and more willing to share knowledge.

(3) Incentives through the establishment of trust. Knowledge sharing requires trust, and trust in turn will promote sharing. The organization members obtained the trust between members through the knowledge sharing, which is also an effective motivator. Knowledge sharing incentive mechanism is only a means, not the end in itself. It is hope that members to participate in knowledge sharing activities autonomy, intrinsic motivation and positive through effective incentive mechanism.

(c) Organizational cultural integration

In the VTIT, cultural integration means that the recognition of different cultures, attention to their differences on the basis of mutual respect, complement and coordinate. You and I become a new organizational culture which has a strong culture of stability and "hybrid" cross-cultural superiority. It supports the effective transfer of knowledge between virtual team members. Cultural characteristics: It is a culture of trust and cooperation. Cultural differences and cooperate to solve scientific and technological innovation within the virtual team members, collaborative knowledge transfer in a recipe. It is a culture of openness. Specific measures include:

(1) To analyze and identify cultural differences

Technological innovation in virtual teams, between partners should respect each other and try to understand the cultural differences between the two sides. Team members should be excluded to maximize the impact of their own cultural values and strive to overcome the "self-referential standards," as far as possible in accordance with the characteristics of each other's culture, customs and practices to determine the problems faced, seeking solutions.

(2))To strengthen the cultural communication and exchange

Cross- cultural communication is the respect and appreciation of science and technology innovation within the VTIT, It allows team members to understand intentions of each other. Encourage extensive and frequent exchanges and communication between members.

(3) To establish the concept of win-win cooperation

Belief of mutually benefit is the basis of cooperation. The sustainable development of relationship is ensured through cooperation. Sharing resources, costs and risks are important reasons for the 
development of virtual technology innovation team. The Union parties are all winners as long as the cooperation is successful. Not only get access to new technology, but also be able to learn from each other and improve their R\&D capabilities. Establishing the concept of win-win cooperation and maintaining the enthusiasm of cooperation lasting alliance parties is benefit to improve the performance of virtual technology innovation team ultimately.

\section{Summary}

Virtual Technology Innovation team is dynamic. The relationship between the members is not static. With the extension of cooperation time, changes happened in the external market. There will be a reasonable for the partnership to make the appropriate adjustments. For example, a small range of areas of cooperation can be expanded or reduced, large areas can be combined to make a new choice in the form of this relationship. Change is abandoning the original relationship, which can stimulate constructive conflict.

This article emphasized on virtual university technology innovation team. But due to the lack of resource, study over high-tech virtual enterprise technology innovation research teams is ignored. Further research is required about whether divergences exist. Virtual technology innovation team association with other research institutions, such as governments, businesses and intermediaries form an external knowledge network. The design of knowledge flow mechanism in the external network is worthy of further study and discussion.

\section{Acknowledgements}

This work supported by Youth Foundation of Mianyang Normal University (2013B11).

\section{References}

[1] X H Wang, B S Zhang. Virtual technology innovation team and the way knowledge flow process [J]. Modern Management Science, 2010 (6):14-16.

[2]P A John, W Peter. Generic Strategy and Performance: An Empirical Test of the Miles and Snow Typology[J]. British Journal of Management, 1993, 4(1): 29-36.

[3] Y S Ji, Z Y Zhang. Dynamic mechanism of technological innovation system design[J]. Technical Economics and Management, 2009 (2): 23-27.

[4] M S Granovetter. Economic action and social structure: The problem of embeddedness[J]. American Journal of Sociology, 1985,91: 481-510.

[5] M S Granovetter. The strength of weak ties[J]. American Journal of Sociology, 1973(78):1360-1380.

[6]R I Sutton, B M Straw. Research in organizational behavior[M]. C T Greenwich: JAI Press, 2000: 345-423.

[7]W M Cohen, D A Levinthal. Absorptive capacity: a new perspective on learning and innovation[J]. Administrative science Quarterly, 1990(35): 128-152.

[8] R Reed, R J DeFillippi. Casual ambiguity, barriers to imitation and sustainable competitive advantage[J]. Academy of Management Review, 1990(15): 88-102. 